\title{
Powered Stapler Malfunction During Laparoscopic Nephrectomy
}

\author{
Shieh Ling Bang, MBChB, MRCS, MMed, YuYi Yeow, MBBS, MRCS, \\ Keng Siang Png, MBBS, MRCS, MMed, FRCS \\ Department of Urology, Tan Tock Seng Hospital, Singapore (all authors).
}

\begin{abstract}
Powered laparoscopic staplers have entered the market in the last few years. We report the first case of a malfunction of a powered laparoscopic stapler during a laparoscopic nephrectomy for a nonfunctioning kidney. The complication was salvaged with further laparoscopic maneuvers by the surgeon without conversion or any further intraoperative complication. Powered laparoscopic staplers, like the nonpowered versions, are equally prone to device malfunction despite manual safety override mechanisms. Laparoscopic surgeons using these devices must be aware of this rare complication and possess the necessary skills to troubleshoot and overcome the problem without endangering the patient.
\end{abstract}

Key Words: Laparoscopic surgery, Nephrectomy, Surgical staplers

Citation Bang SL, Yeow Y, Png KS. Powered stapler malfunction during laparoscopic nephrectomy. CRSLS e2014.00290. DOI: 10.4293/CRSLS.2014.00290.

Copyright (c) 2014 SLS This is an open-access article distributed under the terms of the Creative Commons Attribution-Noncommercial-ShareAlike 3.0 Unported license, which permits unrestricted noncommercial use, distribution, and reproduction in any medium, provided the original author and source are credited.

Address correspondence to: Dr. Keng Siang Png, MBBS, MRCS, MMed, FRCS, Department of Urology, Tan Tock Seng Hospital, 11 Jalan Tan Tock Seng, Singapore 308433. Telephone: + 656256 6011, Fax: +656252 7282, E-mail: keng_siang_png@ttsh.com.sg

\section{INTRODUCTION}

Laparoscopic surgery is performed with greater frequency as surgical skills improve and technological advances are made. With this comes the increasing use of laparoscopic instruments. The laparoscopic linear stapler first appeared in clinical use in the early 1990s. The powered laparoscopic linear stapler is a recent development. The Echelon Flex Powered Endopath stapler (Ethicon Endo-Surgery, Inc, Somerville, New Jersey) was launched in late 2011.

There have been some case reports of equipment malfunctions with the use of laparoscopic staplers during surgery. Deng et $\mathrm{al}^{1}$ reported a $1 \%$ failure rate during urologic laparoscopy. In laparoscopic nephrectomy, Chan et $\mathrm{al}^{2}$ reported a $1.7 \%$ malfunction rate, with an open conversion rate of $20 \%$. Not all equipment failures are reported, and the true incidence of adverse events is probably unknown. A survey conducted by Kwazneski et $\mathrm{al}^{3}$ on minimally invasive program directors showed that $86 \%$ had either personal or peer experience with laparoscopic stapler malfunction, with $27 \%$ noting that it had happened $\geq 3$ times.

Laparoscopic staplers have gained widespread acceptance by most urologists performing laparoscopic ne- phrectomy. Laparoscopic staplers are generally very reliable. Many urologists are now even stapling both the renal artery and vein en bloc without ill effects ${ }^{4-7}$ However, device malfunctions may lead to catastrophic consequences.

Since the powered stapler was introduced in 2011, we reviewed the U.S. Food and Drug Administration's medical device complication database- the MAUDE (Manufacturer and User Facility Device Experience) databasefrom 2009 and found no previous report of malfunction of this device. We report the first case of a powered laparoscopic stapler malfunction during a laparoscopic left nephrectomy.

\section{Case Description}

Our patient was a 71-year-old Chinese woman with history of chronic pelviureteric junction obstruction that resulted in a nonfunctioning, hydronephrotic left kidney. This was complicated with 1 episode of pyonephrosis, which required the insertion of a percutaneous nephrostomy tube. The patient elected to undergo laparoscopic left simple nephrectomy after the episode of sepsis had resolved. 
The patient was prepared and positioned for laparoscopic nephrectomy in a modified right lateral position. Pneumoperitoneum was created using a Veress needle, and the ports were placed under direct vision. A 12-mm trocar for the laparoscope was placed at the umbilicus, and $35-\mathrm{mm}$ trocars were used for dissection and retraction. The colon was mobilized to expose the Gerota fascia. The renal hilum was dissected and prepared for en bloc stapling in the usual fashion. After the renal hilum was dissected free, a 45-mm powered laparoscopic stapler (Powered Echelon Flex) was used to divide both the main artery and the vein en bloc through the umbilical 12-mm trocar while using a 5-mm laparoscope for vision. This occurred smoothly without any mishap. A second $45-\mathrm{mm}$ stapler was reloaded to divide an upper pole branch. After squeezing the firing trigger, the stapling mechanism advanced by only about $1 \mathrm{~cm}$ before stopping abruptly. The knife reverse switch was activated, but this could not reverse the knife. Next, an attempt was made to use the manual override lever. However, the lever was immobile, and thus the knife could not be reversed.

With the stapler stuck in this position in the 12-mm trocar, additional laparoscopic maneuvers were required. First, a new 12-mm trocar was placed at the lateral border of the rectus for the 10-mm laparoscope. Next, the surgeon's left-hand 5-mm trocar was exchanged for a $12-\mathrm{mm}$ trocar. With the stapler still stuck, the upper pole hilar tissue medial to the stapler was dissected with ultrasonic dissection to create space for an additional stapler. An endovascular gastrointestinal anastomosis stapler (Endo-GIA, Covidien, Mansfield, Massachusetts) was inserted through the left-hand 12-mm trocar to divide the tissue medial to the stuck stapler without any bleeding. After the hilum was completely freed, the kidney was dissected off the adrenal gland and mobilized off its lateral attachments.

The powered stapler disengaged after the kidney was free and removed from the body. The patient recovered uneventfully and was discharged on postoperative day 2 .

\section{DISCUSSION}

Powered staplers were introduced in 2011, and they were intended to reduce unwanted movements at the tip of the stapler. Instead of a manual squeezing mechanism, a powered firing mechanism advances the staples. Just like the manual versions, these firing mechanisms are susceptible to device failure. A review of the MAUDE database from 2009 to the present revealed 167 incident reports involving surgical stapler malfunction. ${ }^{8}$
Fifty-eight of these incidents (38\%) were true stapler mechanism failures, and all of them occurred in nonpowered staplers. One incident occurred during a laparoscopic gastric bypass whereby the stapler malfunctioned while creating an anastomosis. The anastomosis was then secured by sutures and interrupted U-clips and there were no further intraoperative incidents after the remedial action. The patient was discharged without any complication. ${ }^{8}$ Another incident occurred during laparoscopic sleeve gastrectomy where the stapler's handles were locked and the surgeon could not open it despite multiple attempts. The surgeon then introduced a new stapler through the supraumbilical port as the locked stapler was in the left mid clavicular port. The new stapler was carefully placed next to the locked one and the stomach was divided, leaving the locked stapler on the part of the stomach to be removed.?

Boggi et al ${ }^{10}$ described another primary endo-GIA stapler failure that occurred during a robotic en bloc resection of liver parenchyma and the right hepatic vein. This operation was undertaken in an obese woman diagnosed with a solitary liver metastasis from a carcinoid tumor. Two forceps were initially applied to the vena cave below the injury for hemostasis. A third robotic arm performed a laparoscopic repair of the caval with interrupted polypropylene sutures. The patient was subsequently transfused with 2 units of packed red blood cells and recovered uneventfully.

A review of the techniques used to secure the renal hilum during laparoscopic donor nephrectomy by Hsi et al ${ }^{11}$ reported that 59 out of the 92 (64\%) complications that occurred were related to failures of endoscopic staplers. The most common mechanisms of stapler failure were missing or malformed staple lines (51\%). This is followed by the failure of the stapler to release, making up $25 \%$ of the cases. Intraoperative conversions to open surgery were required for 21 patients $(36 \%)$, and the estimated overall failure rate for staplers was 3.0\%.

A review by Chan et $\mathrm{al}^{2}$ of 565 patients who underwent laparoscopic nephrectomy reported 10 cases of stapler malfunction (1.7\%). Eight of these cases involved the renal veins, and open conversions to repair the hilum were necessary in 2 cases (20\%). The etiology of the failure was primary instrument failure in 3 cases and preventable causes in the remaining 7 cases.

In this report, we described the first case in the literature of a malfunction of a powered laparoscopic stapler during laparoscopic nephrectomy. This occurred at the critical step of hilar control. The built-in manual over- 
ride corrective mechanisms in the device also failed in our case. Fortunately, the main artery and vein had already been divided, leaving a small upper pole artery. Being a nonfunctional kidney, the arterial inflow from this upper pole branch was probably low, preventing venous congestion and troublesome back bleeding from the kidney. With the patient being stable and no catastrophic bleeding, the surgeon elected to employ additional laparoscopic maneuvers without resorting to conversion to open surgery. In an event where troublesome bleeding occurs, another option would be to place a laparoscopic Satinsky clamp to arrest the bleeding before conversion to open surgery.

The techniques used to salvage a malfunctioning powered laparoscopic stapler are similar to those used to salvage a standard stapler. Laparoscopic surgeons must be aware of the recovery maneuvers in new instruments, failing which, they should be able to perform additional laparoscopic maneuvers or convert to open surgery.

\section{References:}

1. Deng DY, Meng MV, Nguyen HT, Bellman GC, Toller ML. Laparoscopic linear cutting stapler failure. Urology. 2002;60(3): 415-419.

2. Chan D, Bishoff JT, Ratner L, Kavoussi LR, Jarret TW. Endovascular gastrointestinal stapler device malfunction during laparoscopic nephrectomy: early recognition and management. J Urol. 2000;164(2):319-321.

3. Kwazneski D 2nd, Six C, Stahlfeld K. The unacknowledged incidence of laparoscopic stapler malfunction. Surg Endosc. 2013;27(1):86-89.
4. Buse S, Gilfrich C, Pfitzenmaier J, Bedke J, Haferkamp A, Hohenfellner M. En bloc stapler ligation of the renal vascular pedicle during laparoscopic nephrectomy. BJU Int. 2008;101(7): 878-882.

5. Ou CH, Yang WH, Tzai TS. En bloc stapling of renal hilum during hand-assisted retroperitoneoscopic nephroureterectomy in dialysis patients. Urology. 2008;72(3):589-592.

6. Rapp DE, Orvieto MA, Gerber GS, Johnston WK 3rd, Wolf JS Jr, Shalhav AL. En bloc stapling of renal hilum during laparoscopic nephrectomy and nephroureterectomy. Urology. 2004; 64(4):655-659.

7. Kouba E, Smith AM, Derksen JE, Gunn K, Wallen E, Pruthi RS. Efficacy and safety of en bloc ligation of renal hilum during laparoscopic nephrectomy. Urology. 2007;69(2):226-229.

8. U.S. Food and Drug Administration. Manufacturer and User Facility Device Experience Database (MAUDE). http://www. accessdata.fda.gov/scripts/cdrh/cfdocs/CFMAUDE/search.CFM. Accessed June 28, 2007.

9. ElGeidie A, Gadel Hak N, Abdulla T. Stapler's malfunction during laparoscopic sleeve gastrectomy: an unusual but correctable complication. Surg Obes Relat Dis. 2013;9(1):144-146.

10. Boggi U, Moretto C, Vistoli F, D'Imporzano S, Mosca F. Robotic suture of a large caval injury caused by endo-GIA stapler malfunction during laparoscopic wedge resection of liver segments VII and VIII en-bloc with the right hepatic vein. Minim Invasive Ther Allied Technol. 2009;18(5):306-310.

11. Hsi RS, Ojogho ON, Baldwin DD. Analysis of techniques to secure the renal hilum during laparoscopic donor nephrectomy: review of the FDA database. Urology. 2009;74(1):142-147. 